\title{
Prevenção e remediação das dificuldades de aprendizagem: adaptação do modelo de resposta à intervenção em uma amostra brasileira
}

\author{
ROSELAINE PONTES DE ALMEIDA \\ Universidade Presbiteriana Mackenzie, São Paulo, SP, Brasil \\ CAROLINA JULIEN MATTAR DE TOLEDO PIZA \\ Universidade Federal de São Paulo, São Paulo, SP, Brasil \\ THIAGO DA SILVA GUSMÃO CARDOSO \\ Universidade Federal de São Paulo, São Paulo, SP, Brasil \\ MÔNICA CAROLINA MIRANDA \\ Universidade Federal de São Paulo, São Paulo, SP, Brasil
}

RESUMO

O objetivo deste estudo foi adaptar e colocar em prática no contexto brasileiro um dos modelos mais aceitos de prevenção e remediação das dificuldades de aprendizagem, a resposta à intervenção (RTI). A pesquisa foi realizada em duas etapas: i) adaptação e implementação da camada 1 , intervenção coletiva instrucional, na qual participaram duas professoras de primeiro ano do ensino fundamental que aplicaram a RTI em sala de aula; ii) desenvolvimento e implementação da camada 2 , intervenção no grupo-alvo que permaneceu com desempenho abaixo da média no estudo 1. Participaram dez crianças e a intervenção ocorreu três vezes por semana, com duração de cerca de quarenta minutos cada. Todas as atividades tiveram como foco estratégias pedagógicas e interventivas baseadas nas habilidades cognitivas relacionadas à leitura. Os resultados do presente estudo mostram que esse pode ser um modelo adequado ao contexto brasileiro, sendo uma alternativa para avaliar e intervir nas necessidades dos alunos.

PALAVRAS-CHAVE

dificuldade de aprendizagem; prevenção; remediação; resposta à intervenção. 
PREVENTION AND REMEDIATION OF LEARNING DIFFICULTIES: ADAPTATION OF RESPONSE TO AN INTERVENTION MODEL IN A BRAZILIAN SAMPLE

\begin{abstract}
The aim of this study was to adapt and implement a prevention and remediation model of learning disabilities, the Response to Intervention model (RTI), in a Brazilian context. The research was conducted in two stages: i) the adaptation and implementation of tier 1, with a collective instructional intervention, undertaken by two elementary 1st year teachers, who implemented the RTI in their classroom; ii) development and implementation of tier 2, with the intervention target group that remained with a below average performance in stage 1 of the present study. A total of ten children participated and the intervention took place in forty minutes sessions, three times a week. All activities focused on teaching strategies and on underpinning cognitive skills related to reading. Results indicated that the RTI model is appropriate for the Brazilian context, acting as an alternative form of assesment and intervention for the student's needs.
\end{abstract}

KEYWORDS

learning difficulties; prevention; remediation; response to intervention.

\title{
PREVENCIÓN Y REMEDIOS A LAS DISCAPACIDADES DE APRENDIZAJE: ADAPTACIÓN DEL MODELO DE RESPUESTA A LA INTERVENCIÓN EN UNA MUESTRA BRASILEÑA
}

\section{RESUMEN}

El objetivo de este estudio fue adaptar y poner en práctica al contexto brasileño uno de los modelos de mayor aceptación de prevención y remedios a las dificultades de aprendizaje: la respuesta a la intervención (RTI). La investigación se realizó en dos etapas: i) adaptación y aplicación de la capa 1, intervención colectiva de instrucción, en la que participaron dos maestras del $1^{\text {o }}$ de primaria, que aplicaron la RTI en el aula; ii) desarrollo e aplicación de la capa 2, intervención en el grupo blanco, que permaneció con desempeño por abajo del promedio en el estudio 1. Participaron diez niños, y la intervención ocurrió tres veces a la semana, con una duración de cerca de cuarenta minutos cada una. Todas las actividades se han centrado en estrategias pedagógicas y de intervención basadas en las habilidades cognitivas relacionadas con la lectura. Los resultados de este estudio muestran que ese puede ser un modelo apropiado al contexto brasileño, siendo una alternativa para evaluar e intervenir en las necesidades de los alumnos.

PALABRAS CLAVE

dificultad de aprendizaje; prevención; remedio; respuesta a la intervención. 


\section{INTRODUÇÃO}

Dificuldades de aprendizagem é um termo genérico que abarca um grupo heterogêneo de problemas capazes de alterar as possibilidades de aprendizagem de uma pessoa, influenciados por fatores relacionados à escola, à família e ao próprio aprendiz (Rotta, 2006). Ocorrem durante a aquisição ou desenvolvimento de competências e podem atrasar ou reduzir sua consecução (Capovilla, 2011). Nesse sentido, é importante identificar a origem do problema, para que medidas que visem a minimizá-los e até extingui-los possam ser adotadas o mais precocemente possível.

A literatura tem apontado a prevenção como um fator de extrema importância no combate às dificuldades de aprendizagem, uma vez que há grandes chances de sucesso nos casos em que a dificuldade da criança é precocemente identificada, além da presença de maior neuroplasticidade nos primeiros anos da infância, ou seja, a maior adaptação funcional ou estrutural que permite a aquisição do conhecimento (Casella; Amaro Junior; Da Costa, 2011).

Apesar desse reconhecimento, no Brasil o modelo usual de ensino espera a criança falhar para intervir, dando pouca ênfase à prevenção e identificação precoce. Em outros contextos, onde essas recomendações são postas em prática, um dos modelos de intervenção em nível preventivo que tem demonstrado melhor eficácia para os estudantes são os programas baseados na resposta à intervenção (Batsche et al., 2005).

A resposta à intervenção (do inglês response to intervention), também conhecida pela sigla RTI, é definida como um processo de tomada de decisões de ensino baseado no sucesso ou falha do estudante em situação de risco durante a intervenção especializada (Strangeman et al., 2006). A RTI permite identificar precocemente crianças que apresentam problemas acadêmicos e comportamentais, monitorar o progresso das crianças com risco para desenvolver dificuldades nessas áreas e ofertar intervenções cada vez mais intensivas, baseadas na resposta do próprio estudante (Fletcher et al., 2009).

Modelos interventivos dessa natureza caracterizam-se por considerar que estudantes que não recebem instrução abordando suas necessidades logo no início da escolarização estão sujeitos a desenvolver dificuldades acadêmicas acentuadas. Assim, recomenda-se monitorar o desempenho escolar dos estudantes como forma de auxiliar a identificação daqueles que não apresentam avanços, permitindo a colocação em prática ou redirecionamento de intervenções mais precocemente (idem).

Essa abordagem tem orientado decisões educacionais, incluindo decisões sobre a eficácia e intensidade da instrução e intervenção, elegibilidade para os programas de apoio ao ensino e a elaboração de programas educativos diferenciados (Batsche et al., 2005). O modelo, valendo-se desses pressupostos, propõe três níveis (camadas) de intervenção, que dependem da resposta do estudante à instrução, como demonstrado na Figura 1.

A intervenção na camada 1 tem um caráter instrucional, ocorre três vezes por semana, dura cerca de vinte minutos por dia e abarca, de forma preventiva, todos os estudantes. Nessa fase, são os professores que colocam em prática uma variedade de estratégias de ensino (apoiadas em pesquisa científica) e monitoram 
o desempenho dos estudantes por meio de avaliações que incluem componentes curriculares e comportamentais.

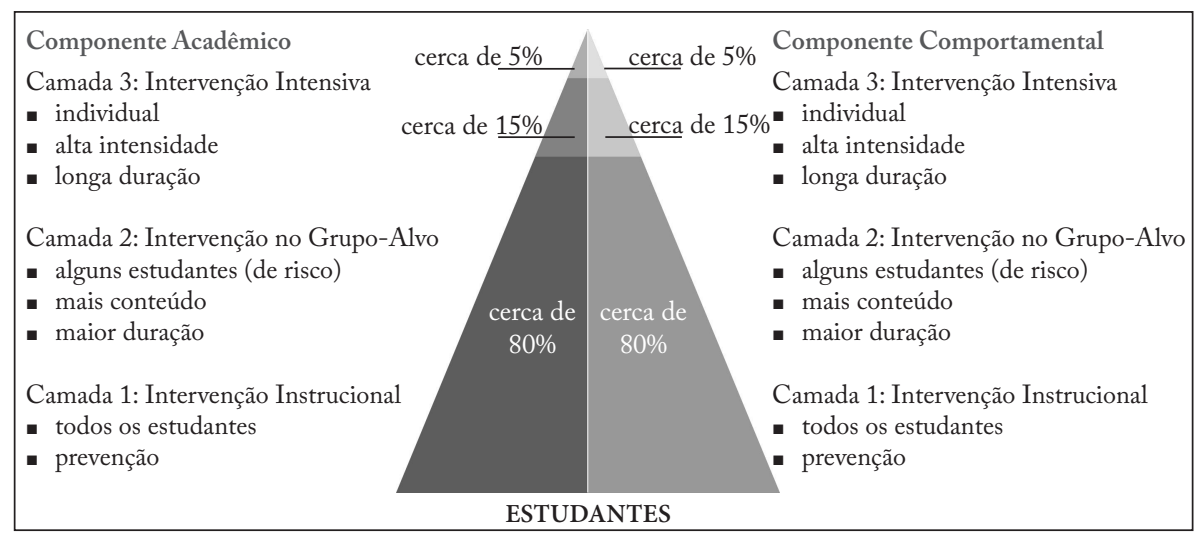

Figura 1 - Modelo de intervenções acadêmicas e comportamentais em níveis cada vez mais intensivos.

Fonte: Fletcher e Vaughn (2009, p. 31). Os percentuais representam estimativas do número de crianças em cada nível.

Na camada 2 a intervenção é remediativa, contínua e direcionada a um grupo-alvo (estudantes de risco), tem duração de quarenta minutos por dia e ocorre três vezes por semana. É ofertada por um profissional de apoio e direcionada aos alunos cujo desempenho e taxa de progresso encontram-se abaixo da média de seus pares da mesma sala de aula, escola ou distrito. $\mathrm{O}$ progresso do estudante é monitorado com frequência para determinar a eficácia da intervenção; a avaliação sistemática é realizada para determinar a fidelidade ou a integridade com que a instrução e as intervenções estão sendo colocadas em prática.

$\mathrm{Na}$ camada 3, a intervenção é individual e intensiva, tem duração de quarenta e cinco a sessenta minutos por dia e ocorre cinco vezes por semana, com monitoramento semanal ou quinzenal do desempenho dos estudantes. É ofertada por um especialista que, com uma equipe multidisciplinar, utiliza múltiplas fontes de dados (pais, professores) para avaliar a criança e determinar a elegibilidade para a educação especial ou serviços relacionados.

Desde sua criação, a RTI tem sido recomendada por alguns pesquisadores (por exemplo, Fuchs; Fuchs, 2006; Fletcher et al., 2009), uma vez que tem sido um modelo sugerido como um meio de reduzir os encaminhamentos a especialistas, fornecendo instruções bem desenhadas e intervenções de alta qualidade na educação geral (National Joint Committee on Learning Disabilities - NJCLD, 2005).

Outra razão importante para o aumento do interesse pela RTI é a abundância de pesquisas recentes sobre leitura, que vêm demonstrando que programas interventivos bem desenhados resultam em melhorias significativas para a maioria dos alunos com dificuldades nessa área do conhecimento (idem). Assim, a RTI tem o aval de muitos pesquisadores e organizações profissionais e governamentais (Fletcher et al., 2009; Fuchs et al., 2003), pois seu objetivo de melhorar o desempenho de todos os alunos e obter melhores resultados comportamentais em crianças com 
dificuldade de aprendizagem tem representado mudanças nas práticas educacionais nos contextos em que tem sido colocada em prática.

Considerando que a RTI é um modelo que pode proporcionar a oportunidade de estudantes com dificuldades serem identificados e cuidadosamente monitorados, sendo ainda uma abordagem capaz de melhorar a aprendizagem no ensino geral, reduzir os encaminhamentos para profissionais especializados (NRCLD, 2007), o objetivo deste trabalho foi realizar e adaptar as camadas 1 e 2 ao contexto educacional brasileiro.

\section{MÉTODO}

A fim de alcançar o objetivo proposto, o presente trabalho foi desenvolvido em duas fases sucessivas, amplamente descritas a seguir.

\section{ETAPA I - ADAPTAÇÃO E IMPLEMENTAÇÃO DA CAMADA 1 DA RTI}

\section{Métodos e procedimentos}

Este estudo foi realizado em duas fases: i) adaptação do modelo com a formação das professoras de primeiro ano do ensino fundamental e ii) colocação em prática da camada 1 da RTI pelas professoras em suas salas de aula.

Fase 1: Diante do objetivo de adequar a RTI à realidade do sistema público de ensino brasileiro, priorizou-se a promoção da reestruturação didática das aulas, com vistas a novas práticas pedagógicas, embasadas cientificamente. Participaram duas professoras de primeiro ano do ensino fundamental, que realizaram uma formação inicial. Essa formação ocorreu em quatro encontros de cerca de duas horas cada e compreendeu: a) esclarecimentos e discussões de conceitos e estratégias pedagógicas; b) elaboração e execução de atividades práticas envolvendo habilidades relacionadas à leitura - nesse caso, consciência fonológica, vocabulário e reconhecimento de letras; c) reestruturação didática das aulas, com vistas a novas práticas pedagógicas, baseadas em instruções sistemáticas e explícitas (correspondente à explicitação, exposição e uso das formas linguísticas das letras do alfabeto na leitura e escrita).

A reestruturação didática das aulas foi voltada à alfabetização e visou a práticas pedagógicas baseadas nas habilidades de leitura, incluindo a manipulação mental de elementos linguísticos correspondentes à consciência fonológica (consciência de palavras, de sílabas, de fonemas, rimas e aliterações), ampliação do vocabulário, reconhecimento de letras e desenvolvimento de habilidades lexicais. Algumas atividades foram elaboradas para esse fim, e outras foram adaptadas de Capovilla e Capovilla (2007), Micheletti et al. (2002), Marques et al. (2001), Baqués (2006a; 2006b) e Santos, Navas e Pereira (1997). As atividades são disponibilizadas na íntegra na dissertação de mestrado da pesquisadora principal do presente artigo (Almeida, 2012).

Fase 2: Realização do modelo RTI em sala de aula. Refere-se aos objetivos da camada 1 do modelo, em que todos os alunos regularmente matriculados beneficiam-se da instrução. Assim, as duas professoras aplicaram a RTI em suas salas de aula. Houve, desse modo, a participação de 51 crianças: 26 alunos da Turma 
A (14 meninas e 12 meninos), com média de idade de 6,4 anos ( $\mathrm{DP}=0,49)$, e 25 alunos da Turma B (11 meninas e 14 meninos), com média de idade de 6,2 anos $(\mathrm{DP}=0,28)$.

A avaliação por meio de medidas de desempenho (que permitem o monitoramento do progresso dos estudantes participantes), nessa fase do estudo, foi realizada antes e após o período de intervenção por meio dos seguintes instrumentos:

1) Avaliação de leitura: foi aplicada aos alunos a versão brasileira do The reading decision test (RDT) (Pinheiro, 2011) - subtestes de leitura de letras e de palavras, nos quais são apresentadas letras reais e formas inventadas, palavras reais e palavras inventadas (pseudopalavras), respectivamente, sendo solicitado à criança que faça a distinção. A correção é feita de acordo com o número total de respostas corretas. Essa tarefa durou aproximadamente dez minutos e foi realizada coletivamente, em apenas uma sessão, no horário regular de aula das crianças. Apesar de a escala ter sido desenvolvida para ser aplicada pelo professor, no presente estudo foi aplicada pela pesquisadora.

2) Avaliação do desempenho dos alunos: as professoras responderam ao Protocolo de Caracterização de Desempenho Escolar dos Alunos (PCDEA), ${ }^{1}$ um questionário que investiga o desempenho acadêmico dos estudantes e os classifica em "muito ruim", "ruim", "suficiente", "bom" e "muito bom" em itens referentes à leitura, escrita, linguagem e matemática. $\mathrm{O}$ preenchimento do protocolo ocorreu no final do primeiro semestre letivo, após o período de intervenção e simultaneamente à avaliação das crianças.

Todos os procedimentos do presente estudo foram aprovados pelo Comitê de Ética em Pesquisa da Universidade Federal de São Paulo (UNIFESP) (protocolo n. 003/2011). A representante da instituição, as professoras, os pais ou responsáveis pelas crianças e as próprias crianças assinaram os termos de consentimento autorizando a aplicação dos procedimentos da pesquisa.

\section{Análise dos dados}

Foram realizadas análises qualitativas verificando as adaptações necessárias à colocação em prática do modelo em relação à amostra selecionada e às fases de desenvolvimento do estudo. Análises quanto à adequação do modelo, bem como às dificuldades e/ ou facilidades do processo, também foram realizadas, baseadas nos relatos das professoras.

$\mathrm{Na}$ análise quantitativa, caracterizou-se o desempenho de todos os alunos nos testes de leitura de letras e de leitura de palavras do RDT, considerando escores brutos, por meio do cálculo de $z$-scores e gráficos antes e após a realização da

1 Elaborado pela doutora Pascale Engel de Abreu e colaboradores do Grupo de Pesquisa em Memória Operacional, Aprendizado e Efeitos Ambientais da Universidade de Luxemburgo, em parceria com a Universidade Federal de São Paulo. Instrumento não comercializado. 
intervenção instrucional. Também foi feita análise de frequência para os resultados do PCDEA. A análise quantitativa dos dados foi realizada por meio do Statistic Package for the Social Science (SPSS) para Windows (Versão 20, SPSS Inc. ${ }^{\odot}$, Chicago, Illinois).

\section{Resultados}

Para que a RTI fosse colocada em prática no Brasil, algumas adaptações fizeram-se necessárias, considerando as especificidades de nossa realidade socioeconômica e de nosso sistema de ensino. $\mathrm{O}$ Quadro 1 apresenta as principais adaptações realizadas na amostra brasileira.

Quadro 1 - Comparação modelo original de RTI e adaptações da camada 1 na amostra brasileira

\begin{tabular}{|l|l|}
\hline \multicolumn{1}{|c|}{ Modelo original de RTI (EUA) } & \multicolumn{1}{c|}{ Adaptaç̃̃es na amostra brasileira } \\
\hline Rastreio universal & $\begin{array}{l}\text { Rastreio em duas salas de primeiro } \\
\text { ano do ensino fundamental }\end{array}$ \\
\hline Triagem universal do comportamento & Não houve \\
\hline Monitoramento periódico do desempenho & Monitoramento antes e após as intervenções \\
\hline
\end{tabular}

Fonte: Banco de dados da pesquisa.

Elaboração dos autores.

A caracterização do desempenho no RDT das 51 crianças participantes está demonstrada na Figura 2. Comparando os dois momentos de avaliação, foi possível observar que, na etapa prévia à realização da intervenção, 9 alunos da Turma A e 13 alunos da Turma B apresentaram desempenho próximo a menos $1(-1)$ desvio-padrão em relação à média esperada no RDT (Pinheiro, 2011). Na etapa pós-intervenção, observamos que esses índices foram reduzidos, tanto na Turma A $(n=4)$ quanto na Turma B $(n=4)$, indicando que muitas crianças avançaram, passando de uma zona de risco para uma zona de normalidade.

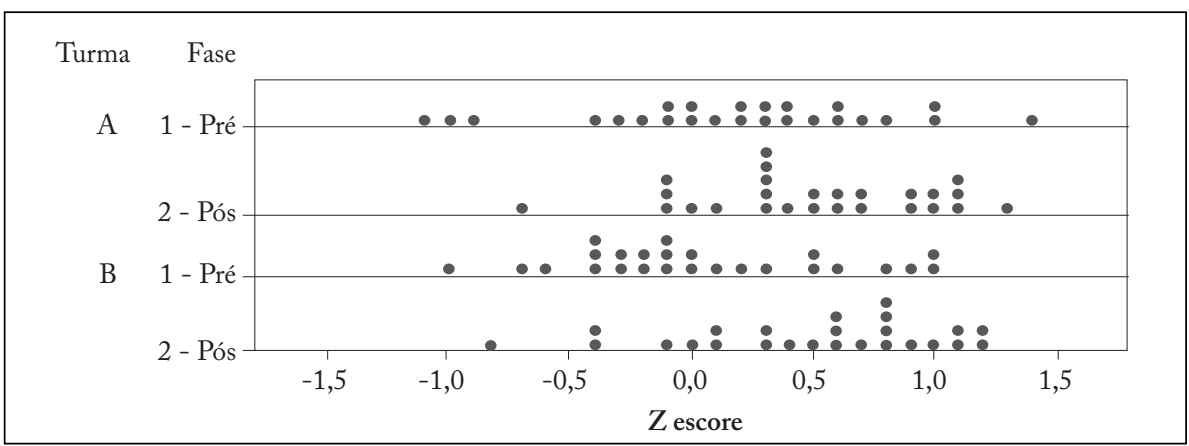

Figura 2 - Desempenho das crianças nas avaliações pré e pós-intervenção do subteste de leitura de letras e palavras do RDT no estudo 1.

Fonte: Almeida (2012, p. 51). 
Já no subteste de leitura de palavras, verificamos que houve maior concentração de estudantes da Turma $\mathrm{A}(\mathrm{n}=7)$ nos índices próximos a menos um $(-1)$ desvio-padrão, enquanto na Turma $B(n=1)$ esse índice foi menor durante a etapa pré-intervenção. Já na pós-intervenção, observamos que esse índice foi reduzido na Turma $A(n=2)$ e foi nulo na Turma B $(n=0)$.

No que se refere às classificações dos alunos no PCDEA (Quadro 2), verificou-se que, em leitura, 23,06\% ( $n=6)$ dos estudantes da Turma A obtiveram classificações ruim e muito ruim em decodificação, e 19,22\% ( $n=5)$, em compreensão. $\mathrm{Na}$ Turma B esses índices foram de $12,0 \%(\mathrm{n}=3)$ tanto para decodificação quanto para compreensão.

\section{Quadro 2 - Classificação do desempenho acadêmico das crianças no PCDEA no estudo 1}

\begin{tabular}{|c|c|c|c|c|c|c|c|c|c|c|c|}
\hline & & \multicolumn{2}{|c|}{ Leitura } & \multicolumn{3}{|c|}{ Escrita } & \multicolumn{2}{|c|}{$\begin{array}{l}\text { Linguagem } \\
\text { oral }\end{array}$} & \multicolumn{3}{|c|}{ Matemática } \\
\hline 营 & 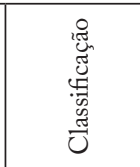 & 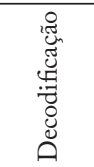 & 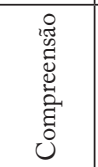 & 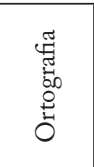 & 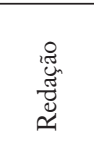 & 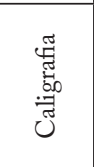 & 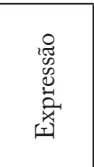 & 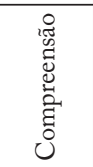 & 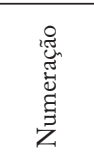 & 怤 & 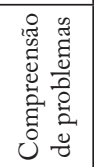 \\
\hline A & Muito ruim & $\begin{array}{r}11,53 \% \\
(n=3)\end{array}$ & $\begin{array}{r}11,53 \% \\
(n=3)\end{array}$ & $\begin{array}{r}3,85 \% \\
(n=1)\end{array}$ & $\begin{array}{r}23,08 \% \\
(n=6)\end{array}$ & $\begin{array}{r}3,85 \% \\
(n=1)\end{array}$ & $\begin{array}{r}11,53 \% \\
(n=3)\end{array}$ & $\begin{array}{r}11,53 \% \\
(n=3)\end{array}$ & $\begin{array}{r}0 \% \\
(\mathrm{n}=0)\end{array}$ & $\begin{array}{r}3,84 \% \\
(n=1)\end{array}$ & $\begin{array}{r}3,84 \% \\
(\mathrm{n}=1)\end{array}$ \\
\hline & Ruim & $\begin{array}{r}11,53 \% \\
(\mathrm{n}=3)\end{array}$ & $\begin{array}{r}7,69 \% \\
(\mathrm{n}=2)\end{array}$ & $\begin{array}{r}7,69 \% \\
(\mathrm{n}=2)\end{array}$ & $\begin{array}{r}0 \% \\
(\mathrm{n}=0)\end{array}$ & $\begin{array}{r}7,69 \% \\
(\mathrm{n}=2)\end{array}$ & $\begin{array}{r}7,69 \% \\
(\mathrm{n}=2)\end{array}$ & $\begin{array}{r}7,69 \% \\
(\mathrm{n}=2)\end{array}$ & $\begin{array}{r}7,69 \% \\
(\mathrm{n}=2)\end{array}$ & $\begin{array}{r}7,69 \% \\
(\mathrm{n}=2)\end{array}$ & $\begin{array}{r}7,69 \% \\
(\mathrm{n}=2)\end{array}$ \\
\hline & Suficiente & $\begin{array}{r}11,53 \% \\
(n=3)\end{array}$ & $\begin{array}{r}11,53 \% \\
(\mathrm{n}=3)\end{array}$ & $\begin{array}{r}15,38 \% \\
(n=4)\end{array}$ & $\begin{array}{r}30,77 \% \\
(\mathrm{n}=8)\end{array}$ & $\begin{array}{r}15,38 \% \\
(n=4)\end{array}$ & $\begin{array}{r}3,84 \% \\
(n=1)\end{array}$ & $\begin{array}{r}15,38 \% \\
(n=4)\end{array}$ & $\begin{array}{r}15,38 \% \\
(n=4)\end{array}$ & $\begin{array}{r}19,23 \% \\
(n=5)\end{array}$ & $\begin{array}{r}26,92 \% \\
(n=7)\end{array}$ \\
\hline & Bom & $\begin{array}{r}11,53 \% \\
(\mathrm{n}=3)\end{array}$ & $\begin{array}{r}11,53 \% \\
(\mathrm{n}=3)\end{array}$ & $\begin{array}{r}34,61 \% \\
(\mathrm{n}=9)\end{array}$ & $\begin{array}{r}46,15 \% \\
(n=12)\end{array}$ & $\begin{array}{r}30,77 \% \\
(\mathrm{n}=8)\end{array}$ & $\begin{array}{r}46,15 \% \\
(\mathrm{n}=12)\end{array}$ & $\begin{array}{r}46,15 \% \\
(n=12)\end{array}$ & $\begin{array}{r}53,85 \% \\
(n=14)\end{array}$ & $\begin{array}{r}50 \% \\
(\mathrm{n}=13)\end{array}$ & $\begin{array}{r}57,69 \% \\
(\mathrm{n}=15)\end{array}$ \\
\hline & Muito bom & $\begin{array}{r}11,53 \% \\
(n=3)\end{array}$ & $\begin{array}{r}11,53 \% \\
(n=3)\end{array}$ & $\begin{array}{r}38,46 \% \\
(n=10)\end{array}$ & $\begin{array}{r}0 \% \\
(\mathrm{n}=0)\end{array}$ & $\begin{array}{l}42,3 \% \\
(n=11)\end{array}$ & $\begin{array}{r}23,07 \% \\
(n=6)\end{array}$ & $\begin{array}{r}30,76 \% \\
(\mathrm{n}=8)\end{array}$ & $\begin{array}{r}23,07 \% \\
(n=6)\end{array}$ & $\begin{array}{r}19,23 \% \\
(n=5)\end{array}$ & $\begin{array}{r}0 \% \\
(\mathrm{n}=0)\end{array}$ \\
\hline B & Muito ruim & $\begin{array}{r}4 \% \\
(n=1)\end{array}$ & $\begin{array}{r}4 \% \\
(n=1)\end{array}$ & $\begin{array}{r}4 \% \\
(n=1)\end{array}$ & $\begin{array}{r}4 \% \\
(n=1)\end{array}$ & $\begin{array}{r}4 \% \\
(n=1)\end{array}$ & $\begin{array}{r}8 \% \\
(n=2)\end{array}$ & $\begin{array}{r}0 \% \\
(\mathrm{n}=0)\end{array}$ & $\begin{array}{r}0 \% \\
(\mathrm{n}=0)\end{array}$ & $\begin{array}{r}4 \% \\
(n=1)\end{array}$ & $\begin{array}{r}4 \% \\
(n=1)\end{array}$ \\
\hline & Ruim & $\begin{array}{r}8 \% \\
(n=2)\end{array}$ & $\begin{array}{r}8 \% \\
(n=2)\end{array}$ & $\begin{array}{r}8 \% \\
(n=2)\end{array}$ & $\begin{array}{r}8 \% \\
(n=2)\end{array}$ & $\begin{array}{r}4 \% \\
(n=1)\end{array}$ & $\begin{array}{r}4 \% \\
(n=1)\end{array}$ & $\begin{array}{r}12 \% \\
(n=3)\end{array}$ & $\begin{array}{r}8 \% \\
(n=2)\end{array}$ & $\begin{array}{r}4 \% \\
(n=1)\end{array}$ & $\begin{array}{r}8 \% \\
(n=2)\end{array}$ \\
\hline & Suficiente & $\begin{array}{r}36 \% \\
(n=9)\end{array}$ & $\begin{array}{r}28 \% \\
(n=7)\end{array}$ & $\begin{array}{r}8 \% \\
(n=2)\end{array}$ & $\begin{array}{r}8 \% \\
(n=2)\end{array}$ & $\begin{array}{r}12 \% \\
(n=3)\end{array}$ & $\begin{array}{r}12 \% \\
(n=3)\end{array}$ & $\begin{array}{r}4 \% \\
(n=1)\end{array}$ & $\begin{array}{r}8 \% \\
(n=2)\end{array}$ & $\begin{array}{r}20 \% \\
(n=5)\end{array}$ & $\begin{array}{r}20 \% \\
(n=5)\end{array}$ \\
\hline & Bom & $\begin{array}{r}40 \% \\
(\mathrm{n}=10)\end{array}$ & $\begin{array}{r}56 \% \\
(\mathrm{n}=14)\end{array}$ & $\begin{array}{r}40 \% \\
(n=10)\end{array}$ & $\begin{array}{r}40 \% \\
(n=10)\end{array}$ & $\begin{array}{r}32 \% \\
(n=8)\end{array}$ & $\begin{array}{r}48 \% \\
(\mathrm{n}=12)\end{array}$ & $\begin{array}{r}52 \% \\
(\mathrm{n}=13)\end{array}$ & $\begin{array}{r}76 \% \\
(n=19)\end{array}$ & $\begin{array}{r}60 \% \\
(\mathrm{n}=15)\end{array}$ & $\begin{array}{r}64 \% \\
(n=16)\end{array}$ \\
\hline & Muito bom & $\begin{array}{l}12 \% \\
(n=3)\end{array}$ & $\begin{array}{r}4 \% \\
(n=1)\end{array}$ & $\begin{array}{r}40 \% \\
(n=10)\end{array}$ & $\begin{array}{r}0 \% \\
(\mathrm{n}=0)\end{array}$ & $\begin{array}{r}48 \% \\
(n=12)\end{array}$ & $\begin{array}{r}28 \% \\
(n=7)\end{array}$ & $\begin{array}{r}28 \% \\
(n=7)\end{array}$ & $\begin{array}{r}8 \% \\
(n=2)\end{array}$ & $\begin{array}{r}12 \% \\
(n=3)\end{array}$ & $\begin{array}{r}4 \% \\
(n=1)\end{array}$ \\
\hline
\end{tabular}

Fonte: Almeida (2012, p. 52).

Nas habilidades de escrita, os alunos da Turma A obtiveram classificação ruim ou muito ruim em todos os itens avaliados, sendo $11,54 \%(\mathrm{n}=3)$ em ortografia e caligrafia, e 23,08\% ( $n=6)$ em redação. Na Turma B, 12\% ( $n=3)$ dos estudantes obtiveram essas classificações em ortografia e redação, e $8 \%(n=2)$ em caligrafia. 
Em relação às habilidades de matemática, as classificações ruim e muito ruim foram atribuídas a 7,69\% $(\mathrm{n}=2)$ dos alunos em numeração, 11,53\% (n = 3) em contas e 15,4\% ( $n=4)$ em compreensão de problemas, na Turma A; já na Turma B, essas classificações corresponderam a $8 \%(n=2)$ em numeração e contas e $12 \%$ $(n=3)$ em compreensão de problemas.

No que concerne às habilidades de linguagem oral, na Turma A a classificação ruim ou muito ruim foi verificada em 19,22\% $(\mathrm{n}=5)$ dos estudantes tanto nas habilidades de expressão quanto de compreensão; na Turma B, $12 \%(n=3)$ dos estudantes obtiveram essas mesmas classificações nas duas habilidades.

\section{Discussão}

Considerando as adaptações necessárias à colocação em prática da RTI no Brasil, seguimos as recomendações do NJCLD. No documento, a primeira consideração prévia à realização da RTI é em relação ao tempo, no sentido de haver reflexão sobre os ajustes diários necessários na sala de aula para que a intervenção aconteça, além da periodicidade da avaliação da resposta do estudante, bem como o próprio período estipulado para cada nível de intervenção. Há que se considerar também o regimento institucional, para que formações de professores e reuniões da equipe colaboradora sejam incluídas no calendário escolar. Para atender a essa recomendação, foi elaborado e posteriormente apresentado à equipe gestora da escola um cronograma de atividades, prevendo os objetivos e detalhando cada fase do projeto.

Outros aspectos importantes da colocação em prática bem-sucedida da RTI dizem respeito à separação de um espaço na escola para avaliações individuais ou em pequenos grupos e à disponibilização de suporte financeiro para aquisição de materiais necessários às intervenções, incluindo dispositivos tecnológicos e recursos humanos (contratação de terapeutas para a camada 3, por exemplo). Em nosso estudo, os recursos físicos foram disponibilizados pela equipe escolar, e parte do material de intervenção foi coberto pela cota semanal de cópias a que cada professora tinha direito.

No que se refere às dificuldades ou facilidades do processo, por meio do relato das professoras observamos que houve envolvimento, interesse, aceitação e participação das docentes em todas as fases desse estudo. Ressalta-se, porém, que acolheram o projeto como uma "solução para o trabalho no primeiro ano", uma vez que, segundo informações de uma das professoras, não havia orientações bem definidas quanto à obrigatoriedade ou não de alfabetizar os alunos nessa etapa escolar.

Para a realização da RTI em sala de aula, ressalta-se que, independentemente da forma como a escola colocou isso em prática, o modelo deve seguir uma estrutura que inclui: a) o rastreio universal dos estudantes; b) níveis de intervenções; c) monitoramento do progresso dos alunos; e d) intervenções baseadas em dados curriculares e de pesquisa (Fuchs; Fuchs, 2005; Mellard; Johnson, 2007). Assim, no que tange às adaptações realizadas durante a presente pesquisa, estas foram elaboradas considerando as especificidades do sistema de educação brasileiro e visando preservar minimamente a estrutura básica do modelo.

Como já mencionado, no modelo original de RTI o rastreio dos estudantes é universal, assim é possível determinar quais alunos estão "em risco" por não se 
encontrarem na média de seus pares de mesma idade e nível de escolaridade. Em nosso estudo, ainda que a triagem não tenha sido realizada em todas as classes da escola, esta foi feita com todos os estudantes das duas salas participantes.

Há recomendação ainda de que a intervenção em todos os níveis seja permeada por avaliações de monitoramento - um processo pelo qual as práticas de instrução são periodicamente avaliadas e ajustadas com base em dados da resposta do estudante a avaliações breves, de rápida aplicação e fácil manuseio (Johnson-Gros, 2008). Em nosso estudo, isso não foi realizado devido aos objetivos de adaptação do modelo, e não à análise de sua eficácia. Johnson-Gros (idem) pontua que, para os professores, os dados provenientes dessas avaliações auxiliam na determinação de mudanças em currículos, materiais ou procedimentos instrucionais. Em razão da adaptação, essa revisão didática não ocorreu, e as professoras não tiveram parâmetros específicos para autoavaliação, recebendo apenas o feedback da pesquisadora a respeito de sua atuação.

De qualquer forma, no modelo original, a avaliação pode ser baseada no desempenho no final da intervenção, no crescimento ao longo da intervenção, ou em ambos (Fuchs, 2003). No presente estudo, essas diretrizes foram consideradas, de modo que incluímos na avaliação instrumentos padronizados que oferecem medidas de habilidades de leitura e escrita.

Também importante, ainda foi utilizado o RDT, um instrumento que avalia a aquisição das habilidades básicas de leitura de maneira rápida, eficaz e com baixo custo, que pode ser administrado coletivamente por educadores e, como afirma Pinheiro (2011), proporciona o estabelecimento de medidas de detecção e intervenção precoce, diminuindo os impactos causados no desenvolvimento da criança.

Como observado, os resultados obtidos na pós-intervenção dos dois subtestes do RDT demonstrou melhora de desempenho dos estudantes das duas turmas. Podemos supor, a partir desses resultados, que as crianças avançaram no reconhecimento de letras em decorrência do treino em relação a isso, ocorrido durante o período de intervenção. Entretanto, esse resultado também pode ser interpretado como evidência de validade relacionada a mudanças do desenvolvimento nos sujeitos avaliados (Anastasi; Urbina, 2000).

As classificações de desempenho dos estudantes nas áreas de leitura, escrita, matemática e linguagem oral, analisadas com base no PCDEA, permitiram identificar as crianças que apresentavam dificuldades nessas habilidades. Observamos que, com exceção de duas crianças (um aluno da Turma A e um aluno da Turma B), as quais ficaram com pontuação menos um (-1) desvio-padrão no RDT, todos os outros alunos que obtiveram as piores classificações nas habilidades avaliadas por meio do PCDEA foram os mesmos que apresentaram desempenho rebaixado nos testes. Esse dado está de acordo com a afirmação de Mellard, McKnight e Woods (2009), que pontuam que a avaliação dos educadores contribui na decisão sobre se os alunos necessitam ou não de algum nível de intervenção, pois esclarecem se o estudante está ou não acompanhando o currículo. Isso ainda indicou que os resultados nas avaliações padronizadas não se distanciam muito da percepção geral que o professor tem a respeito do desempenho de seus alunos.

Assim, apesar da extrema relevância dos instrumentos padronizados, há recentes discussões sobre a avaliação ser um processo e que este possa abarcar a 
avaliação na educação e a avaliação da educação (Didonet, 2001), que "a avaliação será sempre da criança em relação a si mesma e não comparativamente com as outras crianças", o que já é preconizado pelo RTI no monitoramento.

Os resultados desse estudo corroboram dados da literatura que sugerem que o risco de uma criança apresentar dificuldades na leitura e escrita pode ser reduzido quando instruções sistemáticas, de alta qualidade, envolvendo habilidades de consciência fonológica, reconhecimento de palavras e vocabulário, são fornecidas pelo professor em sala de aula (Torgesen, 2002; Compton et al., 2010).

Nesse sentido, concordamos com Fuchs (2003) quando afirma que avaliar a capacidade de resposta à instrução em um contexto da sala de aula tem a vantagem de oferecer um quadro normativo no próprio ambiente de avaliação, ou seja, a capacidade de resposta de um aluno pode ser comparada com o padrão médio de respostas dos demais estudantes daquela classe.

Por fim, vale ressaltar que nos Estados Unidos cada escola deve ter uma equipe de apoio a professores, cuja função é informar ao docente as referências de cada aluno dentro dos primeiros vinte dias do ano letivo, acompanhar a colocação em prática da RTI e fornecer feedback aos docentes sobre sua atuação, enfocando as principais problemáticas observadas e fornecendo um plano de melhoria das ações em sala de aula (Johnson-Gros, 2008). É importante considerar que essa rede de apoio favorece a realização da RTI de forma mais estruturada e eficaz, sendo uma importante recomendação para que o modelo possa ser futuramente posto em prática em larga escala no Brasil.

\section{ETAPA II - ADAPTAÇÃO E REALIZAÇÃO DA CAMADA 2 DA RTI}

\section{Métodos e procedimentos}

Nesse estudo foi realizada a colocação em prática e a adaptação da camada 2 do modelo RTI, que se destina à oferta de intervenção suplementar aos alunos que não responderam à intervenção da camada 1 . Assim, participaram 10 alunos, sendo 6 da Turma A ( 2 meninas e 4 meninos) e 4 da Turma B (todos meninos), participantes do estudo 1. A média de idade dos alunos da Turma $\mathrm{A}$ foi de 6,6 anos $(\mathrm{DP}=0,56)$, e a da Turma $\mathrm{B}$ foi de 6,5 anos $(\mathrm{DP}=0,46)$.

Todas as crianças participantes desse estudo realizaram avaliações antes e após o período de intervenção com os seguintes instrumentos:

1) Provas de avaliação dos processos de leitura - PROLEC (Capellini; Oliveira; Cuetos, 2010). Os subtestes aplicados foram:

Nome ou som das letras: foram apresentados às crianças vinte estímulos (letras isoladas), para que elas os nomeassem ou dissessem o seu som.

Leitura de palavras e pseudopalavras: foi apresentada às crianças uma lista de sessenta estímulos (vinte palavras de alta frequência, vinte palavras de baixa frequência e vinte pseudopalavras), para que elas as lessem.

Igual-diferente em palavras e pseudopalavras: foram apresentados às crianças vinte pares de estímulos (metade palavras e a outra metade pseudopalavras), para que elas dissessem se os estímulos eram iguais ou diferentes. 
Os escores são computados de acordo com o número de respostas corretas. Em todos os subtestes foi considerada a pontuação total da criança.

2) Consciência fonológica: instrumento de avaliação sequencial - $\mathrm{CON}$ FIAS (Moojen et al., 2007). As duas partes do instrumento foram aplicadas: consciência de sílabas e consciência fonêmica. Os escores são calculados de acordo com o número de respostas corretas para cada subitem: sílaba, fonema e total.

\section{Sistematização da intervenção}

Após a avaliação, foi colocada em prática a camada 2, sendo as crianças divididas em dois grupos de intervenção: um grupo com 6 crianças (Turma A) e outro com 4 crianças (Turma B).

A intervenção suplementar foi composta de quatro blocos de atividades, que incluíram: o treino explícito e progressivo de atividades de reconhecimento de letras (bloco 1), consciência fonológica (bloco 2), vocabulário (bloco 3), percepção, atenção e memória (bloco 4), sendo os alunos estimulados três vezes por semana, durante cerca de quarenta minutos por dia. Algumas atividades estão descritas a seguir.

Bloco 1 - Atividades táteis e sinestésicas com letras: fornecemos às crianças letras móveis, massa de modelar, cola colorida e letras em alto relevo, para que reconhecessem as que compunham seus nomes e os escrevessem utilizando os materiais.

Bloco 2 - Consciência fonológica:

Consciência de palavras: foram realizadas atividades com segmentação de sentenças em palavras e verificação do comprimento de palavras, substituição de palavras em frases e substituição de pseudopalavras por palavras.

Rima: foram realizadas atividades visando à produção oral de palavras que rimassem, identificação de figuras cujos nomes rimassem e classificação de figuras conforme a terminação de seus nomes falados.

Aliteração: foram realizadas atividades com produção oral de palavras que aliterassem e escolha de figuras cujos nomes aliterassem com um modelo.

Consciência silábica: foram realizadas atividades de marcação de sílabas em canções, síntese silábica, contagem de sílabas em nomes próprios, contagem de sílabas durante a nomeação oral de figuras e classificação conforme seu comprimento.

Identificação e consciência fonêmica: foram realizadas atividades visando à identificação do fonema inicial de palavras para classificação de figuras, escolha de figuras cujos nomes começassem com o mesmo som de uma figura-modelo e substituição de vogais em palavras.

Bloco 3 - Vocabulário: composto de atividades de nomeação de objetos e figuras, associação de ideias por categorias, atribuição de diferentes significados de uma mesma palavra, partes que formam o todo e descoberta de palavras por meio de pistas contextuais.

Bloco 4 - Percepção, atenção e memória: composto de atividades de atenção seletiva, identificação semântica, identificação de semelhanças e diferenças 
entre objetos, atividades de fechamento, jogo dos sete erros, tarefas de percepção figura-fundo e atividades mnemônicas.

Todas essas atividades também estão disponíveis na íntegra na dissertação de mestrado da pesquisadora (Almeida, 2012).

As atividades foram realizadas coletivamente, em duplas e em pequenos grupos, com as crianças sentadas em cadeiras, em forma de círculo, ou em pé. Mesmo nas atividades coletivas, todos os alunos eram solicitados a participar.

Todas as intervenções ocorreram na biblioteca da escola, durante o período das aulas, nos meses de agosto, setembro, outubro e início de novembro de 2011. O caráter lúdico das atividades e os materiais de apoio utilizados foram selecionados para despertar o interesse e a motivação das crianças.

\section{Análise dos dados}

Realizou-se análise qualitativa, verificando as adaptações necessárias à colocação em prática da camada 2 da RTI em relação à amostra selecionada e às fases de desenvolvimento do estudo. $\mathrm{Na}$ análise quantitativa, realizou-se verificação do Índice de Mudança Fiel (Reliable Change Index - RCI), significativo para $\alpha=0,05$, $\mathrm{z}>1,96$, a fim de verificar mudanças de desempenho após a intervenção nos subtestes do PROLEC e do CONFIAS. A análise quantitativa dos dados foi realizada por meio do SPSS para Windows (Versão 17.0, SPSS Inc. ${ }^{\odot}$, Chicago, Illinois).

\section{Resultados}

Nesse estudo, assim como no estudo I, algumas adaptações foram necessárias para que o modelo RTI fosse posto em prática. As adaptações realizadas ocorreram nos aspectos referentes à estrutura de espaço e tempo das intervenções, assim como nos pressupostos de triagem comportamental e de monitoramento de desempenho dos estudantes. O Quadro 3 apresenta essas adaptações.

O desempenho das crianças nas avaliações pré e pós-intervenção nos subtestes do PROLEC estão representados no Quadro 4. Verificou-se que no subteste "identificação de letras" todas as crianças mostraram escores significativamente melhores após o período de intervenção; o mesmo ocorreu nos resultados do subteste "igual-diferente em palavras e pseudopalavras", exceto para uma criança. Já em relação aos resultados obtidos no subteste "leitura de palavras e pseudopalavras", verificou-se que a maior parte dos estudantes apresentou evolução na avaliação pós-intervenção, com exceção de duas crianças (20\%) que não obtiveram pontuação significativamente maior no reconhecimento de palavras e pseudopalavras, permanecendo com pontuação igual a 0 .

No que concerne à análise do desempenho das crianças em CONFIAS, nota-se que o desempenho dos estudantes melhorou tanto no subteste de fonemas quanto no subteste de sílabas (Quadro 5). Na classificação quanto à hipótese de escrita, verificou-se que $80 \%$ dos participantes $(n=8)$ foram classificados como pré-silábicos, $10 \%(\mathrm{n}=1)$ como silábicos e $10 \%(\mathrm{n}=1)$ como silábico-alfabéticos. Após a intervenção, $50 \%(\mathrm{n}=5)$ dos estudantes avançaram para a hipótese alfabética de escrita, e $30 \%(n=3)$ avançaram para a hipótese silábico-alfabética, indicando 
progresso do nível de escrita, enquanto somente $20 \%(n=2)$ dos estudantes permaneceram na hipótese pré-silábica.

\section{Quadro 3 - Comparação modelo original de RTI e} adaptações da camada 2 na amostra brasileira

\begin{tabular}{|l|l|}
\hline \multicolumn{1}{|c|}{ Modelo original de RTI (EUA) } & \multicolumn{1}{c|}{ Adaptações na amostra brasileira } \\
\hline Aplicada por um profissional de apoio & Aplicada pela pesquisadora \\
\hline Intervenções em cinco dias da semana & Intervenções em três dias da semana \\
\hline Espaço físico específico para intervenção & Intervenção realizada em diferentes espaços \\
\hline Triagem comportamental & Não houve \\
\hline Monitoramento periódico do desempenho & Monitoramento antes e após a intervenção \\
\hline
\end{tabular}

Fonte: Banco de dados da pesquisa.

Elaboração dos autores.

Quadro 4 - Escore bruto nas avaliações pré e pós-intervenção por participante nos subtestes do PROLEC no estudo 2

\begin{tabular}{|r|r|r|c|c|c|c|c|r|r|}
\hline Participante & \multicolumn{3}{|c|}{ Identificação de Letras } & \multicolumn{3}{|c|}{ Igual-diferente } & \multicolumn{3}{|c|}{$\begin{array}{c}\text { Palavras e } \\
\text { pseudopalavras }\end{array}$} \\
\hline & Pré & Pós & RCI & Pré & Pós & RCI & Pré & Pós & \multicolumn{1}{c|}{ RCI } \\
\hline 1 & 6 & 7 & $3,44^{*}$ & 11 & 17 & $14,28^{*}$ & 0 & 0 & 0 \\
\hline 2 & 13 & 17 & $13,7^{*}$ & 16 & 18 & $4,76^{*}$ & 49 & 57 & $8,16^{*}$ \\
\hline 3 & 8 & 17 & $9,18^{*}$ & 15 & 16 & $2,38^{*}$ & 0 & 8 & $8,16^{*}$ \\
\hline 4 & 15 & 17 & $6,89^{*}$ & 13 & 15 & $4,76^{*}$ & 0 & 12 & $12,24^{*}$ \\
\hline 5 & 0 & 3 & $10,34^{*}$ & 9 & 11 & $4,76^{*}$ & 0 & 0 & 0 \\
\hline 6 & 16 & 19 & $10,34^{*}$ & 14 & 19 & $11,9^{*}$ & 41 & 53 & $12,24^{*}$ \\
\hline 7 & 4 & 13 & $31,03^{*}$ & 18 & 19 & $2,38^{*}$ & 0 & 3 & $3,06^{*}$ \\
\hline 8 & 2 & 9 & $24,13^{*}$ & 15 & 17 & $4,76^{*}$ & 0 & 3 & $3,06^{*}$ \\
\hline 9 & 7 & 15 & $27,58^{*}$ & 18 & 18 & 0 & 0 & 9 & $9,18^{*}$ \\
\hline 10 & 3 & 16 & $44,88^{*}$ & 17 & 18 & $2,38^{*}$ & 0 & 4 & $4,08^{*}$ \\
\hline
\end{tabular}

Fonte: Almeida (2012, p. 76).

Obs: RCI (Reliable Change Index), Índice de Mudança Fiel.

* significativo para $\alpha=0,05, \mathrm{z}>1,96$.

\section{Discussão}

Assim como ocorreu na camada 1 , na camada 2 da RTI algumas adaptações fizeram-se necessárias para que o modelo pudesse ser posto em prática no grupo de crianças participantes do estudo. Desse modo, adaptações ocorreram na estrutura de espaço e tempo das intervenções, assim como nos pressupostos de triagem comportamental e de monitoramento de desempenho dos estudantes.

No que se refere ao tempo, considerando que a intervenção proposta nesse estudo ocorre fora do ambiente de sala de aula, a periodicidade desses encontros foi pensada analisando-se a viabilidade da retirada do aluno de suas aulas regulares 
para participar da intervenção suplementar. Sendo assim, essa intervenção, que originalmente é aplicada nos cinco dias letivos da semana e dura aproximadamente quarenta minutos por dia, foi aplicada em três dias da semana, de forma que o tempo estimado de quarenta minutos diários foi preservado.

\section{Quadro 5 - Classificação e escore nas avaliações pré e pós-intervenção} por participante nos subtestes do CONFIAS no estudo 2

\begin{tabular}{|r|c|c|l|c|c|l|}
\hline & Sílaba & Fonema & & Sílaba & Fonema & \\
\hline P* $^{*}$ & Pré & Pré & Classificação & Pós & Pós & Classificação \\
\hline 1 & 15 & 5 & Pré-silábico & 22 & 11 & Pré-silábico \\
\hline 2 & 26 & 10 & Silábico & 39 & 25 & Alfabético \\
\hline 3 & 18 & 9 & Pré-silábico & 32 & 22 & Alfabético \\
\hline 4 & 23 & 3 & Pré-silábico & 35 & 16 & Silábico-alfabético \\
\hline 5 & 18 & 10 & Pré-silábico & 32 & 17 & Silábico-alfabético \\
\hline 6 & 13 & 6 & Pré-silábico & 29 & 25 & Alfabético \\
\hline 7 & 12 & 3 & Pré-silábico & 16 & 9 & Pré-silábico \\
\hline 8 & 30 & 17 & Silábico-alfabético & 37 & 27 & Alfabético \\
\hline 9 & 20 & 8 & Pré-silábico & 32 & 24 & Alfabético \\
\hline 10 & 14 & 9 & Pré-silábico & 28 & 21 & Silábico-alfabético \\
\hline
\end{tabular}

Fonte: Almeida (2012, p. 76).

*P : participante.

Quanto ao espaço para a realização das avaliações e intervenções, o relatório de recomendações para a colocação em prática da RTI, produzido em 2005 pelo NJCLD, explana que a separação de um espaço na escola para tais atividades viabiliza a boa realização do modelo. Contudo, por falta de estrutura física adequada no prédio escolar, não existiu uma sala especialmente reservada para esses fins, sendo necessário realizar as avaliações e intervenções ora na biblioteca da escola, ora na sala de informática.

Do mesmo modo que no estudo I, não ocorreu a triagem comportamental dos estudantes participantes do estudo; também não foram realizadas avaliações periódicas de monitoramento entre os períodos pré e pós-intervenção, sendo considerados como medida de desempenho os resultados dos estudantes no protocolo dos testes aplicados antes e após a intervenção.

Como a intervenção não foi realizada pelas professoras titulares dos alunos, sempre que possível houve troca de informações entre a pesquisadora e as professoras a respeito do desempenho das crianças, tanto na intervenção quanto em sala de aula. A adoção desse tipo de postura é recomendada pelo próprio NJCLD (2005), que considera que parcerias desse tipo favorecem a eficácia do projeto. Entretanto, assim como no estudo I, discussões mais aprofundadas sobre a realização da RTI ficaram inviabilizadas pela ausência da participação ativa de outros membros da equipe escolar nesse processo.

Apesar da análise da eficácia do modelo não ser foco do presente estudo, os resultados obtidos no PROLEC mostraram que a maior parte das crianças avançou 
significativamente nas habilidades avaliadas pelo instrumento após o período de intervenção suplementar. Com base nesse conjunto de dados, podemos presumir que a intervenção contribuiu de forma positiva para elevar o nível dos processos léxicos e de identificação de letras. As crianças que não avançaram foram consideradas com dificuldades na aprendizagem.

Nessa análise, é importante notar que 50\% dos participantes avançaram para a hipótese alfabética de escrita após o período de intervenção suplementar. Esse dado nos mostra que, para metade das crianças, a estratégia alfabética havia sido bem-sucedida, indicando que elas compreenderam a natureza da escrita convencional (Ferreiro; Teberosky, 1991) - um requisito fundamental para a participação autônoma das crianças nos mais diversos contextos de leitura e escrita (Correa, 2011).

Outra observação interessante é que, tanto na avaliação pré quanto na avaliação pós-intervenção, as crianças demonstraram melhor desempenho nas tarefas de consciência silábica em relação às tarefas de consciência fonêmica. Esse dado coaduna-se com a literatura, que aponta que as habilidades de análise fonológica no nível do fonema desenvolvem-se em maior extensão posteriormente à alfabetização (Mousinho; Correa, 2009).

Mousinho e Correa (idem) referem que a consciência silábica é uma habilidade importante para o aprendizado inicial da escrita do português brasileiro. Isso pode ser notado no desempenho das crianças na avaliação pós-intervenção do CONFIAS, na qual os maiores escores em consciência silábica referem-se a classificações em níveis mais elevados quanto à hipótese de escrita.

\section{CONCLUSÕES}

Consideráveis evidências têm demonstrado que utilizar como referência os estudos com foco sobre a neurobiologia da aprendizagem para repensar práticas educacionais e utilizar abordagens consistentes com a ciência cognitiva da leitura, que estejam em consonância com princípios científicos atualizados, são importantes para o estabelecimento de processos mais eficientes de alfabetização (Araújo,2011).

Devido a um quadro heterogêneo de problemas capazes de alterar as possibilidades de aprendizagem de uma pessoa, a identificação precoce e o diagnóstico das dificuldades de aprendizagem constituem-se em processos difíceis, nem sempre precisos, que muitas vezes culminam no encaminhamento de crianças para avaliações e intervenções com profissionais especializados. Embora inúmeros países já reconheçam a necessidade de acompanhamento diferenciado para esses estudantes, no Brasil ainda não há uma política nacional de identificação e acompanhamento dessas crianças, o que torna ainda mais complexo o exercício de intervenção precoce (Navas; Weinstein, 2009).

Em outros contextos, tais problemas têm sido discutidos à luz de modelos de prevenção, cujos resultados de sucesso têm permitido a identificação precoce de crianças da educação infantil e séries iniciais em risco de desenvolverem dificuldade de leitura e que, portanto, requerem atendimento imediato, antes que problemas significativos as impeçam de trilhar o caminho do desenvolvimento normal das habilidades (Mellard; McKnight; Woods, 2009; Compton et al., 2010). Além 
disso, nos Estados Unidos as agências educacionais priorizam a boa formação dos professores, com práticas baseadas em evidências científicas, de forma que esses profissionais estejam preparados para atender a todas as crianças e identificar aquelas que apresentam dificuldades de aprendizagem, sentindo-se preparados para prestar atendimento diferenciado aos alunos que dele necessitarem.

Discussões nesses níveis, ainda que necessárias, são recentes no Brasil. Atualmente não há uma política nacional de prevenção e acompanhamento desses estudantes em nosso país. Mesmo nos Estados Unidos, até alguns anos atrás, a RTI ainda era uma abordagem nova para muitos educadores, pesquisadores e governantes. Por esse motivo, ainda que seja um sistema multicamadas bem integrado (Johnson-Gros, 2008), a RTI configura-se e apresenta-se como um modelo dinâmico, cujos aspectos são modificados de acordo com o regimento interno de cada instituição (Mellard; McKnight; Woods, 2009; Johnson-Gros, 2008) ou de cada contexto específico. É importante ter clareza de que a colocação em prática da RTI em contextos diversos inclui a variação de uma série de fatores, como a seleção e fidelidade das intervenções, as decisões sobre os prazos, os critérios de movimento entre as camadas, bem como recursos destinados às intervenções e à capacitação da equipe escolar. Essas modificações farão com que o modelo varie quanto à flexibilidade e rigidez de certos critérios, o que certamente afetará a possibilidade de generalização e replicação dos resultados do presente estudo.

Contudo, vale ressaltar que a comparação de execução e eficácia da RTI em diferentes abordagens não tem sido explorada, representando um importante e promissor campo de pesquisas (Fuchs; Fuchs, 2006) que pode contribuir trazendo respostas quanto às taxas de sucesso dos alunos após cada nível de intervenção, a padrões ou normas de referência para a realização de cada camada, bem como os instrumentos de rastreio mais sensíveis na identificação dos estudantes com dificuldade.

\section{REFERÊNCIAS}

Almeida, R. P. Prevenção e remediação das dificuldades de aprendizagem: adaptação do modelo de resposta à intervenção numa amostra brasileira. 2012. 104f. Dissertação (Mestrado em Educação e Saúde na Infância e na Adolescência) - Universidade Federal de São Paulo, Guarulhos.

Anastasi, A.; Urbina, S. Testagem psicológica. 7. ed. Porto Alegre: Artes Médicas, 2000. Araújo, A. P. (Coord.). Aprendizagem infantil: uma abordagem da neurociência, economia e psicologia cognitiva. Rio de Janeiro: Academia Brasileira de Ciências, 2011. Baqués, M. Projeto pensamento, ação e inteligência (Projeto PAI - 4). Tradução de Carmem Fales e Cristina M. Oliveira. São Paulo: Edições SM, 2006a.

. Projeto pensamento, ação e inteligência (Projeto PAI - 5). Tradução de Carmem Fales e Cristina M. Oliveira. São Paulo: Edições SM, 2006b.

Batsche, G.M.; Elliott, J.; Graden, J. L.; Grimes, J.; Kovaleski, J. F.; Prasse, D. Response to intervention: policy considerations and implementation. National Association of State Directors of Special Education, 2005. 
Capellini, S. A.; Oliveira, A. M.; Cuetos, F. PROlEC: provas de avaliação dos processos de leitura. São Paulo: Casa do Psicólogo, 2010.

Capovilla, F. C. (Org.). Transtornos de aprendizagem: progressos em avaliação e intervenção preventiva e remediativa. 2. ed. São Paulo: Memnon, 2011.

Capovilla, A. G. S.; Capovilla, F. C. Alfabetização: método fônico. São Paulo: Memnon, 2007.

Casella, E. B.; Amaro Junior, E.; Da Costa, J. C. As bases neurobiológicas da aprendizagem da leitura. In: Araújo, A. P. (Coord.). Aprendizagem infantil: uma abordagem da neurociência, economia e psicologia cognitiva. Rio de Janeiro: Academia Brasileira de Ciências. 2011. p. 37-78.

Compton, D. L.; Fuchs, D.; Fuchs, L. S.; Bryant, J. D. Selecting at-risk first-grade readers for early intervention: eliminating false positives and exploring the promise of a two-stage gated screening process. Journal of Educational Psychology, Washington, DC: American Psychological Association, v. 102, n. 2, p. 327-340, 2010.

Correa, J. A avaliação da escrita da criança: descritores para uma aprendizagem reflexiva. In: CApovilla, F. C. (Org.). Transtornos de aprendizagem 2: da análise laboratorial e da reabilitação clínica para as políticas públicas de prevenção pela via da educação. São Paulo: Memnon, 2011. p. 39-45.

Didonet, V. Creche: a que veio... para onde vai... Em Aberto, Brasília: INEP, v. 18, n. 73, p. 11-27, 2001.

Ferreiro, E.; Teberosky, A. A psicogênese da língua escrita. 4. ed. Porto Alegre: Artes Médicas, 1991.

Fletcher, J. M.; Vaughn, S. Response to intervention: preventing and remediating academic difficulties. Child Development Perspectives, Malden, MA: Journal Customer Services John Wiley \& Sons Inc., v. 3, n. 1, p. 30-37, 2009.

; Lyon, R. G.; Fuchs, L. S.; Barnes, M. A. Transtornos de aprendizagem: da identificação à intervenção. Tradução de Ronaldo Cataldo Costa. Porto Alegre: Artmed, 2009.

Fuchs, D. et al. Responsiveness-to-intervention: definitions, evidence, and implications for the learning disabilities construct. Learning Disabilities Research E Practice, Malden, MA: Journal Customer Services John Wiley \& Sons Inc., v. 18, n. 3, p. 157-171, 2003.

Fuchs, L. S. Assessing intervention responsiveness: conceptual and technical issues. Learning Disabilities Research E Practice, Malden, MA: Journal Customer Services John Wiley \& Sons Inc., v. 18, n. 3, p. 172-186, 2003.

Fuchs, D.; Fuchs, L. S. Responsiveness-to-intervention: a blueprint for practitioners, policymakers, and parents. Teaching Exceptional Children, Thousand Oaks, CA: Sage Publications, v. 38, p. 57-61, 2005.

;___ Introduction to response to intervention: what, why, and how valid is it? Reading Research Quarterly, Malden, MA: Journal Customer Services John Wiley \& Sons Inc., v. 4, p. 93-99, 2006.

Johnson-Gros, K. N. Response to intervention: tier 1. New York: Guilford Press, 2008. Disponível em: <www.gcsd.us/index.php>. Acesso em: 24 jun. 2012. 
Marques, E.; Pellicciotti, T. H.F.; Bombonatto, Q.; Micheletti, C. S. Aprender a compreender: atividades de linguagem e cognição (médio). São Paulo: Plexus, 2001.

Mellard, D. F.; Johnson, E. S. RTI: a practitioner's guide to implementing response to intervention. Thousand Oaks, CA: Corwin Press, 2007.

.; McKnight, M.; Woods, K. Response to intervention screening and progressmonitoring practices in 41 local schools. Learning Disabilities Research \& Practice, Malden, MA: Journal Customer Services John Wiley \& Sons Inc., v. 24, p. 4, p.186-195, 2009.

Micheletti, C. S. et al. Aprender a compreender: atividades de linguagem e cognição. São Paulo: Plexus, 2002.v. I.

Moojen, S. (Coord.); Lamprecht, R.; Santos, R. M.; Freitas, G. M.; Brodacz, R.; Siqueira, M.; Costa, A. C.; Guarda, E. CONFIAS: Consciência Fonológica Instrumento de Avaliação Sequencial. São Paulo: Casa do Psicólogo, 2007.

Mousinho, R.; Correa, J. Habilidades linguístico-cognitivas em leitores e não leitores. Pró-Fono: Revista de Atualização Cientiffica, Barueri: Pró-Fono Departamento Editorial, v. 21, n. 2, p. 113-118, 2009.

Navas, A. L. G.P.; Weinstein, M. A. Distúrbio específico de leitura (dislexia): debates necessários. Revista CEFAC, São Paulo: CEFAC, v. 11, n. 4, p. 545-736, 2009.

NJCLD - National Joint Coomittee on Learning Disabilities. Responsiveness to intervention and learning disabilities. [S.1.: s.n.], 2005. Disponível em: <http://www. ldanatl.org/pdf/rti2005.pdf>. Acesso em: 7 jan. 2012.

NRCLD - National Research Center on Learning Disabilities. What is responsiveness to intervention? [S.1.: s.n.], 2007. Disponível em: <http://www.nrcld.org/ resource_kit/parent/What_is_RTI2007.pdf >. Acesso em: 2 ago. 2011.

Pinheiro, E. C. M.Adaptação do teste de leitura The reading decision test para o português do Brasil em crianças do $1^{\circ}$ ao $5^{\circ}$ ano do Ensino Fundamental. 2011. 117f. Dissertação (Mestrado em Psicobiologia) - Universidade Federal de São Paulo, São Paulo, 2011.

RotтA, N.T. Dificuldades para a aprendizagem. In: ; Ohlweiler, L.; Riesgo, R. S. Transtornos da aprendizagem: abordagem neurobiológica e multidisciplinar. Porto Alegre: Artmed, 2006. p. 113-123.

Santos, M. T. M.; Navas, A. L. G. P.; Pereira, L. D. Estimulando a consciência fonológica. In: Pereira, L. D.; Schochat, E. (Orgs.). Processamento auditivo central: manual de avaliação. São Paulo: Lovise, 1997. p. 85 -90.

Strangeman, N. et al. Response-to-instruction and universal design for learning: how might they intersect in the general education classroom? [S.1.: s.n.], 2006. Disponível em: <http://www.ldonline.org/article/13002>. Acesso em: 11 nov. 2010.

Torgesen, J. K. The prevention of reading difficulties. Journal of School Psychology, Filadélfia, Pensilvânia: Academic Press, n. 40, p. 7-26, 2002. 


\section{SOBRE OS AUTORES}

Roselaine Pontes de Almeida é mestre em educação e saúde na infância e na adolescência pela Universidade Federal de São Paulo (UNIFESP). Professora da Universidade Presbiteriana Mackenzie e da Universidade Paulista (UNIP).

E-mail: roselainepontes@gmail.com

Carolina Julien Mattar de Toledo Piza é mestre em psicobiologia pela Universidade Federal de São Paulo (UNIFESP). Coordenadora técnica do Instituto $\mathrm{ABCD}$.

E-mail: carolatp@gmail.com

Thiago da Silva Gusmão Cardoso é mestre em educação e saúde na infância e na adolescência pela Universidade Federal de São Paulo (UNIFESP). Professor do Centro Universitário Adventista de São Paulo (UNASP).

E-mail: thiago_gusmao1@hotmail.com

Mônica Carolina Miranda é doutora em ciências pela Universidade Federal de São Paulo (UNIFESP). Professora da Universidade Federal do Rio Grande do Norte (UFRN).

E-mail:mirandambr@yahoo.com.br 